'Departamento de Pediatría y Adolescencia, Clínica Las Condes, Santiago, Chile.

${ }^{2}$ Corporación para la Nutrición Infantil (CONIN), Santiago, Chile. ${ }^{3}$ Escuela de Salud Pública, Facultad de Medicina Universidad de Chile.

${ }^{4}$ Departamento Nutrición y Alimentos, Subsecretaría de Salud Pública, Ministerio de Salud, Chile.

${ }^{5}$ Departamento de Pediatría, Universidad de Chile, Campus Norte, Hospital Roberto del Río, Santiago, Chile.

${ }^{6}$ Unidad de Adolescencia Clínica Santa María, Santiago, Chile.

Recibido el 3 de diciembre de 2013, aceptado el 15 de agosto de 2014

Correspondencia a: Dra. Claudia Torrejón Departamento de Pediatría Norte, Universidad de Chile, torrejon_clau@yahoo.com

\section{Mercurio en pescados y su importancia en la salud}

\author{
XIMENA RAIMANN ${ }^{1}$, LORENA RODRÍGUEZ O., ${ }^{2,3}$, \\ PAULINA CHÁVEZ ${ }^{4}$ CLAUDIA TORREJÓN ${ }^{5,6}$
}

\section{Mercury in fish and its importance in health}

The contamination of fish with methylmercury $(\mathrm{MeHg})$ could hamper the health promoting properties of fish. Currently, there is strong evidence about the health benefits of seafood consumption. When consumed by the mother before and during pregnancy it improves neurodevelopment of infants and toddlers. Thereafter it reduces the risk of cardiovascular and other chronic diseases. The benefits of fish are mainly due to its content of omega-3 long chain polyunsaturated fatty acids, including eicosapentaenoic acid (EPA) and docosahexaenoic acid (DHA). Other constituents, such as high biological value proteins, fatsoluble vitamins, minerals and trace elements contribute to the benefits. On the other hand, there is also convincing evidence about the adverse effects of $\mathrm{MeHg}$ on neurodevelopment both during gestation and in early childhood. We herein review the effects of mercury on health. Based on international evidence and new data on the mercury content in Chilean fish, we also propose a recommendation for fish consumption for our population.

(Rev Med Chile 2014; 142: 1174-1180)

Key words: Fish oils; Methylmercury compounds; Recommended dietary allowances.

\section{Mercurio y Salud}

I os desechos de la minería y residuos industriales han dejado un legado de mercurio en vertederos y suelos. Normalmente éste se libera a la atmósfera desde la Tierra a partir de materiales de la corteza natural, incluida la acción volcánica, y procesos antropogénicos como la combustión de carbón y de residuos y la fundición ${ }^{1,2}$.

El mercurio tiene diversos efectos adversos sobre la salud y el medio ambiente; sus compuestos son sumamente tóxicos, especialmente para el sistema nervioso en desarrollo. Su nivel de toxicidad en seres humanos y otros organismos varía según la forma química, cantidad, vía de exposición y vulnerabilidad de la persona expuesta ${ }^{1,2}$.

El mercurio está presente en la mayoría de recursos naturales, incluyendo suelos, carbón, minerales y se presenta en 3 formas: a) Mercurio elemental.

b) Sal inorgánica de mercurio.

c) Mercurio orgánico.

El mercurio orgánico es el de mayor importancia para la salud; sus compuestos incluyen metilmercurio, etilmercurio y fenilmercurio. Todos éstos han sido producidos primariamente como biocidas y pesticidas. El más conocido es el metilmercurio (MeHg), ya que es el compuesto que se encuentra en el ambiente, se deposita en el agua y se acumula en organismos (bioacumulación), concentrándose en las cadenas alimentarias (biomagnificación), especialmente en la cadena alimentaria acuática (peces y mamíferos marinos) siendo luego ingerido por las personas a través de los productos del mar, ${ }^{3,4}$.

La ingesta de MeHg a través de peces y alimentos del mar es actualmente un problema de salud pública, dada su toxicidad en el desarrollo neurológico en fetos y niños ${ }^{5,6}$. 
Mercurio y su importancia en salud - X. Raimann et al

El etilmercurio y el fenilmercurio son usados como bacteriostáticos en varias preparaciones farmacológicas tópicas ${ }^{7}$.

\section{Absorción metabolismo y excreción:}

La mayoría de los compuestos orgánicos de mercurio son absorbidos por ingestión, inhalación y a través de la piel. En general estos compuestos orgánicos son liposolubles y más de $90 \%$ son absorbidos desde el tubo digestivo. Aparecen en la fracción lipídica de la sangre y en el tejido cerebral ${ }^{2,4}$.

El MeHg cruza rápidamente la barrera hematoencefálica y la placenta y su concentración en la sangre fetal es igual o mayor que la de la madre. $\mathrm{Su}$ vida media en sangre es de 40 a 50 días en adultos. Noventa por ciento del $\mathrm{MeHg}$ es excretado a través de la bilis y las heces; un porcentaje menor se excreta en el pelo y la orina. Es posible que se excrete cierta cantidad en la leche, pero en una proporción mucho menor ${ }^{2,4}$.

\section{Toxicidad}

La toxicidad de éstos compuestos orgánicos depende del compuesto, la ruta de exposición y la edad de la persona expuesta ${ }^{2,4}$.

Los signos de toxicidad en la exposición aguda vía digestiva progresan desde parestesias a ataxia y debilidad generalizada, luego disminución de visión y audición, espasticidad muscular, coma y muerte ${ }^{2,4}$.

El $\mathrm{MeHg}$ es un conocido teratógeno en el cerebro del feto, interfiere con la migración neuronal, la organización de núcleos del cerebro y la estratificación de neuronas corticales. En las intoxicaciones con $\mathrm{MeHg}$ procedente de pescados, en Minamata, Japón e Irak, las madres estaban asintomáticas o mostraban efectos tóxicos leves cuando dieron a luz, mientras sus lactantes resultaron severamente afectados, dado que el feto es más susceptible al efecto neurotóxico del MeHg. Si bien estos niños parecían normales al nacer, el retraso psicomotor, la ceguera, sordera y convulsiones se desarrollaron con el paso del tiempo, por lo que los investigadores han intentado también identificar los efectos subclínicos del $\mathrm{MeHg}^{8,9}$.

Ha habido tres extensos estudios, incluidos la cohorte de Irak y dos estudios epidemiológicos prospectivos, uno en las islas Seychelles y otro en las Faroe, los cuales han estudiado los efectos del $\mathrm{MeHg}$ en los niños a diferentes niveles de exposición de sus madres ${ }^{9-11}$. Éstos muestran que la memoria, atención y pruebas de lenguaje se asociaron inversamente con una mayor exposición a $\mathrm{MeHg}$ en los niños de hasta 7 años, incluso después de controlar por otros factores ${ }^{11,12}$.

\section{Marcadores biológicos de exposición mercurio}

La exposición humana al mercurio se evalúa mediante el uso de biomarcadores, que sirven como un sustituto para determinar la dosis interna biológicamente relevante ${ }^{6}$. Los tres biomarcadores principales son: mercurio en sangre, en pelo y en uñas. También puede usarse sangre de cordón umbilical, orina y leche $e^{4,13}$.

\section{Mercurio en sangre total (ST)}

Indica exposición actual o reciente a $\mathrm{MeHg}$. Se correlaciona mejor con la dosis ingerida que la concentración en pelo y alcanza niveles máximos rápidamente (4-14 h). El promedio de vida media de $\mathrm{MeHg}$ en sangre es de 50 días $^{6}$. En poblaciones con patrones frecuentes y regulares de consumo de pescado, el mercurio en ST puede reflejar un estado en equilibrio de la concentración y, por lo tanto, podría ser una medida precisa de la ingesta promedio en el tiempo ${ }^{6,13}$. En poblaciones con consumo infrecuente o irregular, este supuesto estado de equilibrio puede ser inapropiado, y una sola medida podría proporcionar insuficiente información acerca de la magnitud o el período de las exposiciones.

A pesar que el mercurio en ST se correlaciona bien con la exposición a $\mathrm{MeHg}$ en las poblaciones que consumen regularmente pescado, también refleja mercurio inorgánico, que puede provenir de amalgamas dentales y de algunos procesos de desmetilación. Dado que el $\mathrm{MeHg}$ se une de forma preferente a los glóbulos rojos (GR) su medición en GR puede ser un biomarcador más preciso, especialmente en poblaciones con amalgamas dentales y con exposición baja ${ }^{14}$. Sin embargo, los niveles de mercurio en GR y su relación con la ingesta ha sido menos estudiado que en ST y no hay niveles internacionalmente aceptados aún.

Según la OMS, la concentración normal media 
de mercurio en ST oscila entre 5 y $10 \mu \mathrm{g} / \mathrm{L}$ en los individuos sin consumo de pescado contaminado, por su parte el National Research Council (NRC) considera $2 \mu \mathrm{g} / \mathrm{L}$ como la concentración media normal para las poblaciones con poco o ningún consumo de pescado en los Estados Unidos de Norteamérica $^{6,13}$.

\section{Mercurio en pelo}

Este puede indicar la magnitud y el momento de la exposición. La incorporación de mercurio en el folículo del pelo en crecimiento es directamente proporcional a la concentración en sangre, con un retraso de 1-2 meses desde la ingesta de $\mathrm{MeHg}^{6,15}$. La relación entre la ubicación a lo largo de la hebra de cabello y el tiempo de exposición puede ser comprobada suponiendo una tasa constante de crecimiento del pelo de $1,1 \mathrm{~cm}$ por mes ${ }^{6,13}$. Una vez que pasa al pelo, el mercurio no vuelve a la sangre, por lo que constituye un buen marcador de exposición a largo plazo $^{16}$. En las personas que no consumen pescado contaminado, el nivel normal de mercurio en el pelo es de 1-2 ppm (1-2 $\mu \mathrm{g} / \mathrm{g})$, mientras que quienes lo consumen pueden presentar un nivel mayor o igual a $10 \mathrm{ppm}^{4,13}$.

\section{Mercurio en uñas}

Puede ser un indicador del promedio de exposición en el tiempo ${ }^{17-19}$. Sin embargo, la toxicocinética específica de absorción de mercurio desde la sangre hasta el lecho ungular no se conoce bien.

\section{Mercurio en sangre del cordón umbilical}

Este es más preciso que los niveles en el pelo de la madre, a la hora de determinar la exposición prenatal de los niños al MeHg. La concentración en el peso seco del cordón umbilical es casi tan efectiva como la sangre de cordón en la predicción de déficits neuropsicológicos asociados con el MeHg a los 7 años de edad ${ }^{11}$.

La presencia de mercurio en la orina, generalmente representa la exposición al mercurio inorgánico y/o elemental. El mercurio inorgánico se acumula en el riñón y se excreta lentamente a través de la orina. Por lo tanto, los niveles de mercurio en la orina también pueden representar la exposición al mercurio elemental y/o inorgánico que se produjo en algún momento del pasado ${ }^{13}$. En el caso de la leche humana, si bien ésta representa una ruta principal de excreción de sustancias lipofílicas, la mayoría de las formas de mercurio no son lipofílicas. La concentración en la leche humana depende de muchos factores como la edad, masa corporal, tiempo de toma de muestras, estado nutricional, y no se correlacionan con la concentración de mercurio en el pelo de las madres o bebés ${ }^{13}$.

\section{Recomendaciones de consumo de pescado: riesgos versus beneficios}

Los beneficios y riesgos de la ingesta de pescado varían de acuerdo a la especie de pescado, tamaño y formas de cultivo, como también a la cantidad consumida y forma de preparación.

Las mujeres en edad reproductiva, las gestantes, las nodrizas y los niños pequeños son considerados poblaciones sensibles a la exposición de pescados contaminados, dados los efectos negativos en el neurodesarrollo.

En el año 2004 en Estados Unidos de Norteamérica, después de un estudio del contenido de mercurio en sus pescados, un comunicado conjunto entre la Agencia de Protección Ambiental de Estados Unidos de Norteamérica y la Agencia de Alimentos y Medicamentos de Estados Unidos de Norteamérica (FDA) recomendó que las mujeres en edad reproductiva, las gestantes y las nodrizas consumieran $340 \mathrm{~g}$ de pescado a la semana, pero que no consumieran tiburón, pez espada, blanquillo del Golfo de México y caballa, por su alto contenido de mercurio ( $\geq 1 \mathrm{ug} / \mathrm{g}$ de pescado) y limitó a $170 \mathrm{~g}$ el consumo de atún y albacora ${ }^{20}$.

Por otro lado, una publicación de 2007 basada en un estudio longitudinal en el Reino Unido, sugirió que la advertencia de limitar el consumo de pescados y mariscos podría ser deletérea para la salud. El riesgo de no consumir pescado fue estimado como mayor que el riesgo de daño por posibles contaminantes si se consumían sólo 340 $\mathrm{g}$ de pescado semanales ${ }^{21}$.

En el año 2010, en Roma, se realizó una reunión de expertos de la $\mathrm{FAO} / \mathrm{WHO}$ sobre los riesgos y beneficios del consumo de pescado ${ }^{22}$. La tarea para los expertos fue revisar la información de los nutrientes [ácidos grasos omega 3 (AG $\omega-3)$ ] y de contaminantes químicos específicos como el $\mathrm{MeHg}$ en una variedad de peces y comparar los beneficios en la salud (específicamente en el neurodesarrollo infantil y en la prevención de enfermedad cardiovascular) del consumo de pes- 
cado, con los riesgos asociados a la ingesta de los contaminantes del pescado. Así, en base al análisis de la FDA ${ }^{23}$, sobre la relación dosis-respuesta entre el consumo materno de pescado y el coeficiente intelectual (CI) verbal del niño, y estimando que en promedio el consumo de pescado provee 5,4 mg de ácido eicosapentaenoico (EPA) + ácido docosahexaenoico (DHA) por gramo de pescado y 3,6 mg de DHA por gramo (27,8 g de pescado aportan $100 \mathrm{mg}$ de DHA), se encontró que con una ingesta materna de $100 \mathrm{mg}$ de DHA al día, había una ganancia de 2,8 puntos de CI verbal del niño a los 18 meses de vida ${ }^{24}$. En otro trabajo se vio que la ganancia de CI verbal a los 8 años era de 4,2 puntos con la ingesta materna de $100 \mathrm{mg}$ de $\mathrm{DHA}^{21}$. En un tercer trabajo se encontró a los 3 años de vida, una ganancia similar de CI $(3,8$ puntos) con igual ingesta materna de pescado ${ }^{25}$.

En lo que respecta al riesgo cardiovascular en la población, se ha demostrado que la ingesta de $1.750 \mathrm{mg}$ de EPA y DHA a la semana $(250 \mathrm{mg} / \mathrm{d})$, disminuye el riesgo de muerte por enfermedad coronaria ${ }^{26}$, los que estarían contenidos en aproximadamente $320 \mathrm{~g}$ de pescado ${ }^{27}$.

El comité de expertos de la FAO analizó la composición del pescado, desarrollando una matriz que comparaba los niveles de DHA + EPA, con los niveles totales de mercurio en 96 especies de pescado, y los efectos en el CI del niño como resultado del consumo materno de una, dos, cuatro o siete porciones de pescado a la semana (de 100 g cada porción), con diferentes concentraciones de EPA + DHA y MeHg. Los resultados y conclusiones fueron los siguientes:

1) Existe evidencia convincente que el consumo materno de pescado contribuye al óptimo desarrollo neurológico de su hijo.

2) Si se considera la pérdida promedio de CI dada por el mercurio, los riesgos en el neurodesarrollo de no comer pescado exceden a los riesgos de comerlo, incluso con siete porciones de 100 gr a la semana, si los niveles de $\mathrm{MeHg}$ son menores a $1 \mathrm{ug} / \mathrm{g}$.

3) Si se considera la mayor pérdida de CI que puede dar el mercurio, los riesgos en el neurodesarrollo de no comer pescado exceden los riesgos de comerlo incluso comiendo siete porciones de pescado de $100 \mathrm{~g}$ a la semana, siempre y cuando el nivel de $\mathrm{MeHg}$ sea menor a $0,5 \mathrm{ug} / \mathrm{g}$.

4) Si el pescado tiene más de $1 \mathrm{ug} / \mathrm{g}$ de $\mathrm{MeHg}$ los riesgos en el neurodesarrollo de no comer pescado exceden los riesgos de comerlo con hasta dos porciones por semana de pescados que posean más de $8 \mathrm{mg} / \mathrm{g}$ de EPA + DHA.

Las conclusiones finales reportadas por el comité de expertos FAO 2010 fueron que, en la población adulta, el consumo de pescados, especialmente los grasos, disminuye el riesgo de mortalidad por enfermedad coronaria en mujeres en edad reproductiva, gestantes y nodrizas, y optimiza el neurodesarrollo de sus hijos. En los lactantes, niños pequeños y adolescentes, la evidencia es insuficiente para realizar conclusiones cuantitativas de los riesgos y beneficios sobre la salud, sin embargo, el consumo de pescado a esta edad influencia los hábitos dietarios y la salud durante la vida adulta.

En Chile, el límite máximo permitido de concentración de mercurio en pescados y mariscos establecido por el Ministerio de Salud en el Reglamento Sanitario de los Alimentos (RSA), D.S. No 977/96, artículo 160, es 0,5 a $1,5 \mathrm{mg} / \mathrm{kg}$ $(1,5 \mathrm{ug} / \mathrm{g})$ de producto final ${ }^{28}$. Como parte del Programa de Inocuidad de los Alimentos del Ministerio de Salud, durante el año 2012 se diseñó un Plan de Vigilancia Nacional de Metales Pesados en productos del mar, priorizando la vigilancia de mercurio en los productos marinos de mayor extracción y mayor consumo nacional. El tamaño muestral se definió considerando como universo "productos del mar," con un nivel de confianza de $95 \%$ y con una precisión de $5 \%$, resultando un total de 400 muestras $^{29}$. A su vez, este total, se distribuyó en las distintas especies considerando los criterios de mayor extracción, consumo y especies que acumulan mercurio. Del total programado, se tomaron y analizaron 366 muestras. Los muestreos se realizaron en 3 etapas, entre febrero de 2012 y enero de 2013. Los peces incluidos fueron: albacora o pez espada, anchoveta, atún, caballa, congrio colorado, congrio dorado, congrio negro, corvina, jurel, merluza común, merluza de cola, reineta, salmón del Atlántico, salmón plateado, sardina común, sierra, y dos moluscos: almeja y chorito. Del total de 366 muestras, 299 fueron pescados y 67 mariscos. El 99,5\% de las muestras resultaron por debajo de los límites de mercurio total establecidos en el RSA (Tablas 1 y 2).

Los valores encontrados en el total de las muestras (366) están en el rango de 0,01 a 1,57 mg/kg de 
Tabla 1. Límites máximos de concentración de Mercurio en pescados y mariscos (RSA)

\begin{tabular}{|lc|}
\hline & $\begin{array}{c}\text { Límite máximo } \\
(\mathbf{m g} / \mathbf{k g}=\mathbf{u g} / \mathbf{g})\end{array}$ \\
\hline Conservas de pescados y mariscos & 1,0 \\
\hline $\begin{array}{l}\text { Pescado fresco, enfriado y congelado } \\
\text { talla pequeña }\end{array}$ & 0,5 \\
\hline $\begin{array}{l}\text { Pescado fresco, enfriado y congelado } \\
\text { talla grande }\end{array}$ & 1,5 \\
Mariscos frescos & 0,5 \\
\hline
\end{tabular}

Hg total. El promedio general del total de muestras fue de $0,118 \mathrm{mg} / \mathrm{kg}$.

El valor más alto encontrado corresponde a una muestra de albacora, con 1,57 mg/kg, el cual está por sobre el límite máximo de $1,5 \mathrm{mg} / \mathrm{kg}$, superándolo en $5 \%$. La incertidumbre para este valor es de $1,57 \pm 0,22 \mathrm{mg} / \mathrm{kg}$. Esta muestra fue tomada en la Región de Valparaíso, pero su origen es la Provincia de Arauco, Región del Bío-Bío.

El otro resultado que supera la norma vigente, corresponde a una muestra de chorito, con $0,710 \mathrm{mg} / \mathrm{kg}$, el cual está por sobre el límite

Tabla 2. Resultados medición mercurio en pescados y mariscos. Plan de vigilancia nacional de metales pesados en productos del mar, MINSAL 2012

\begin{tabular}{|c|c|c|c|c|c|}
\hline & & Situación por es| & ecie & & \\
\hline Producto & $\stackrel{n}{\text { muestras }}$ & $\begin{array}{c}\text { Regiones } \\
\text { involucradas }\end{array}$ & $\begin{array}{c}\text { Rango } \\
\text { Resultados } \\
\text { (mg/kg) }\end{array}$ & $\begin{array}{c}\text { Promedio } \\
\text { Resultados } \\
\text { (mg/kg) }\end{array}$ & $\begin{array}{c}\text { Norma RSA } \\
\text { (mg/kg) }\end{array}$ \\
\hline Albacora & 19 & II-III-IV-V & $0,280-1,570$ & 0,739 & 1,5 \\
\hline Anchoveta & 11 & $\mid-11$ & $0,010-0,080$ & 0,021 & 0,5 \\
\hline Atún conserva (aceite y agua) & 50 & I-RM & $0,020-0,830$ & 0,212 & 1,0 \\
\hline Besugo & 3 & VIII & $0,070-0,095$ & 0,080 & 0,5 \\
\hline Bilagay & 3 & III & $0,070-0,130$ & 0,110 & 0,5 \\
\hline Caballa & 3 & IV & $0,025-0,180$ & 0,085 & 0,5 \\
\hline Cabrilla & 2 & $\mathrm{XV}$ & $0,180-0,370$ & 0,275 & 0,5 \\
\hline Cojinova & 2 & $\mathrm{XV}$ & $0,040-0,050$ & 0,045 & 0,5 \\
\hline Congrio (Colorado, Dorado y Negro) & 27 & V- VIII-XIV-X-XI-XII & $0,020-0,290$ & 0,117 & 0,5 \\
\hline Corvina & 6 & $|-| X$ & $0,030-0,140$ & 0,056 & 0,5 \\
\hline Jurel & 18 & $|-| I-V \mid I I$ & $0,020-0,050$ & 0,038 & 0,5 \\
\hline Merluza (Común, de Cola) & 56 & V- VII-VIII-X-XI & $0,015-0,260$ & 0,065 & 0,5 \\
\hline Pejerrey & 4 & 1 & $0,020-0,320$ & 0,103 & 0,5 \\
\hline Reineta & 22 & VII-VIII & $0,043-0,185$ & 0,090 & 0,5 \\
\hline Róbalo & 8 & $\mathrm{XIV}-\mathrm{X}$ & $0,028-0,140$ & 0,076 & 0,5 \\
\hline Rollizo & 3 & III & $0,090-0,120$ & 0,107 & 0,5 \\
\hline Salmón (Atlántico y Plateado) & 40 & XIV-X-XI-XII & $0,020-0,130$ & 0,034 & 0,5 \\
\hline Sardina & 4 & XIV-X & $0,025-0,118$ & 0,067 & 0,5 \\
\hline Sierra & 18 & V-VIII-XIV-X & $0,030-0,220$ & 0,066 & 0,5 \\
\hline Almeja & 22 & $X V-V-V I I I-X-X I$ & $0,010-0,110$ & 0,041 & 0,5 \\
\hline Chorito & 39 & II-V-XIV-X & $0,010-0,710$ & 0,054 & 0,5 \\
\hline Choros & 6 & $X V-X$ & $0,010-0,060$ & 0,025 & 0,5 \\
\hline Total & 366 & & $0,010-1,570$ & 0,118 & \\
\hline
\end{tabular}


máximo de $0,5 \mathrm{mg} / \mathrm{kg}$, superándolo en $42 \%$. La incertidumbre para este valor es de 0,71 $\pm 0,10$ $\mathrm{mg} / \mathrm{kg}$. Esta muestra fue tomada en la Región de Los Lagos y su lugar de extracción es también la $\mathrm{X}$ Región. Cabe indicar que se tomaron 39 muestras de choritos, de las cuales 27 corresponden a la Región de Los Lagos, y 26 resultados de esta región son $\leq 0,80 \mathrm{mg} / \mathrm{kg}$.

En términos de promedio, la especie con resultados más elevados es la albacora con $0,739 \mathrm{mg} /$ $\mathrm{kg}$, seguido de la cabrilla $0,275 \mathrm{mg} / \mathrm{kg}$ y el atún en conserva con $0,212 \mathrm{mg} / \mathrm{kg}$. Es importante señalar que para el caso de la cabrilla, sólo se tomaron 2 muestras.

Para el caso del atún se tomaron 50 muestras, 33 en aceite y 17 al agua. El 100\% de las muestras están bajo el valor límite $1,0 \mathrm{mg} / \mathrm{kg}$ del RSA. Este muestreo contempló 19 marcas y 2 países de origen, Ecuador y Tailandia.

Adecuando las unidades de medida a las publicaciones extranjeras existentes, para el contenido de mercurio, todos nuestros pescados están con niveles menores a $1 \mathrm{ug} / \mathrm{g}$, salvo la albacora $(1,57 \mathrm{ug} / \mathrm{g})$.

FAO recomienda para análisis de riesgos y beneficios del consumo de pescado, conocer su concentración de $A G \omega$-3; en Chile prácticamente no existen estudios al respecto, salvo algunos datos experimentales (datos no publicados) en que se encontró que la mayoría de los pescados analizados tendría niveles de DHA + EPA superiores a $8 \mathrm{mg} / \mathrm{g}$ (Tabla 3 ).

Tabla 3. Contenido de DHA + EPA en peces, por gramo de producto* (Adaptado de Valenzuela)

\begin{tabular}{|lc|}
\hline & EPA + DHA $(\mathbf{m g} / \mathbf{g})$ \\
\hline Anchoa & 14 \\
Atún & 17 \\
Bacalao & 2,3 \\
Corvina & 3,5 \\
Jurel & 20 \\
Salmón & 13 \\
Sardina & 14 \\
Trucha** & 9 \\
\hline
\end{tabular}

*Valores promedio. Varían con la época de captura. **Pez de cultivo.

\section{Conclusiones}

Teniendo en cuenta toda la información presentada, la recomendación de consumo para nuestra población incluyendo mujeres en etapa reproductiva, gestantes y nodrizas, no debería requerir restricciones, ya que se podrían consumir hasta siete porciones de $100 \mathrm{~g}$ a la semana $(700 \mathrm{~g})$ de cualquier pescado excepto albacora, $\mathrm{y}$ sigue habiendo un beneficio para la salud. Con lo anterior debería cubrirse además el requerimiento de AG $\omega-3$, aunque aún falta información del contenido de este nutriente en nuestros productos marinos y del nivel de consumo de pescados en nuestra población. Cabe recordar que las nuevas Guías Alimentarias del Ministerio de Salud (2013) proponen el consumo de pescado dos veces a la semana.

Los desafíos para nuestro país serán revisar las Guías Alimentarias del MINSAL, difundir esta información a la población, realizar estudios para conocer el consumo de pescados en Chile, $y$ su composición lipídica, y mantener un sistema de vigilancia y control del contenido de mercurio en los pescados, actualizando periódicamente estos límites.

\section{Referencias}

1. Clarkson TW. The toxicology of mercury. Crit Rev Clin Lab Sci 1997; 34 (4): 369-403.

2. Goldman L, Shannon M, Committee on Environmental Health. Technical Report: Mercury in the Environment: Implications for Pediatricians. Pediatrics 2001; 108 (1): 197-205.

3. Gotelli CA, Astolfi E, Cox C, Cernichiari E, Clarkson TW. Early biochemical effects of an organic mercury fungicide on infants: "dose makes the poison." Science 1985; 227 (4687): 638-40.

4. Programa de las Naciones Unidas para el medio ambiente. Evaluación mundial sobre el mercurio. Publicado por el PNUMA Productos Químicos. Ginebra, Suiza. Diciembre de 2002.

5. Swain EB, Jakus PM, Rice G, Lupi F, Maxson PA, Pacyna $\mathrm{JM}$, et al. Socioeconomic consequences of mercury use and pollution. Ambio 2007; 36 (1): 45-61.

6. NRC (National Research Council). Toxicological Effects of Methylmercury. Washington, DC: National Academy Press. 2000. p 31-71.

7. American Academy of Pediatrics, Committee on Infectious Diseases and Committee on Environmental 
Health. Thimerosal in vaccines-aninterim report to clinicians. Pediatrics 1999; 104 (3 Pt 1): 570-57.

8. Amin-Zaki L, Elhassani S, Majeed MA, Clarkson TW, Doherty RA, Greenwood M. Intra-uterine methylmercury poisoning in Iraq. Pediatrics 1974; 54 (4): 587-95.

9. Bakir F, Damluji SF, Amin-Zaki L, et al. Methylmercury poisoning in Iraq. Science 1973;181 (4096):230-41.

10. Amin-Zaki L, Majeed MA, Elhassani SB, Clarkson TW, Greenwood MR, Doherty RA. Prenatal methylmercury poisoning. Clinical observations over five years. Am J Dis Child 1979; 133 (2): 172-7.

11. Grandjean P, Weihe P, White RF, Debes F, Araki S, Yokoyama Ket al. Cognitive deficit in 7-year-old children with prenatal exposure to methylmercury. Neurotoxicol Teratol 1997; 19 (6): 417-28.

12. Davidson PW, Myers GJ, Cox C, Axtell C, Shamlaye C, Sloane-Reeves J, et al. Effects of prenatal and postnatal methylmercury exposure from fish consumption on neurodevelopment: outcomes at 66 months of age in the Seychelles Child Development Study. JAMA 1998; 280 (6): 701-7.

13. OMS y PNUMA. 2008. Guidance for Identifying Populations at Risk from Mercury Exposure. Ginebra (Suiza).

14. Berglund M, Lind B, Bjömerg KA, Palm B, Einarsson Ö, Vahter M. Inter-individual variation of human mercury exposure biomarkers: a cross-sectional assessment. Environmental Health 4:20; doi:10.1186/1476-069X-4-20 [Online 3 October 2005].

15. Budtz-Jørgensen E, Grandjean P, Jørgensen PJ, Weihe P, Keiding N. Association between mercury concentrations in blood and hair in methylmercury-exposed subjects at different ages. Environ Res 2004; 95 (3): 385-93.

16. Ohba T, Kurokawa N, Nakai K, Shimada M, Suzuki K, Sugawara N, et al.Permanent waving does not change mercury concentration in the proximal segment of hair close to scalp. Tohoku J Exp Med 2008; 214 (1): 69-78.

17. Rees JR, Sturup S, Chen C, Folt C, Karagas MR. Toenail mercury and dietary fish consumption. J Expo Science Environ Epidemiol 2007; 17 (1): 25-30.

18. Garland M, Morris JS, Rosner BA, Stampfer MJ, Spate VL, Baskett CJ, et al. Toenail trace element levels as biomarkers: reproducibility over a 6-year period. Cancer Epidemiol Biomarkers Prev 1993; 2 (5): 493-7.

19. Ohno T, Sakamoto M, Kurosawa T, Dakeishi M, Iwata T, Murata K. Total mercury levels in hair, toenail, and urine among women free from occupational exposure and their relations to renal tubular function. Environ Res 2007; 103 (2): 191-7.
20. USEPA, USFDA 2004. What you need to know about mercury in fish and shellfish. Advice for women who might become pregnant, nursing mothers, young children. Unites States Environmental Protection Agency and United States Food and Drug Administration. Disponible en :www.water.epa.gov/scitech/swguidance /fishshellfish/ [consultado 24 de mayo de 2004fish_ MethylmercuryBrochure].

21. Hibbeln JR, Davis JM, Steer CP, Emmett I, Rogers C, Williams $\mathrm{C}$ et al. Maternal seafood consumption in pregnancy and neurodevelopmental outcomes in childhood (ALSPAC study): an observational cohort study. Lancet 2007; 369 (9561): 578-85.

22. Report of the Joint FAO/WHO Expert Consultation on the Risks and Benefits of Fish Consumption. Rome, Food and Agriculture Organization of the United Nations; Geneva, World Health Organization, 2011. p50 .

23. USFDA 2009. Report of quantitative risk and benefit assessment of consumption of commercial fish. Focusing on fetal neurodevelopment effects (measured by verbal development in children) and on coronary heart disease and stroke in the general population. Draft report. U.S. Department of Health and Human Services, Food and Drug Administration Disponible en: wwww.fda. gov/FoodSafety/ProductSpecificInformation7Seafood/ FoodbornePathogensContaminants/Methylmercury/ ucm088758.htm

24. Daniels JL, Longnecker MP, Rowland AS, Golding J. Fish intake during pregnancy and early cognitive development of offspring. Epidemiology 2004; 15 (4): 394-402.

25. Oken E, Radesky JS, Wright RO, Bellinger DC, Amarasiriwardena CJ, Kleinman KP. Maternal fish intake during pregnancy, blood mercury levels, and child cognition at age 3 years in a US cohort. American Journal of Epidemiology 2008a; 167 (10): 1171-81.

26. Mozaffarian D, Rimm EB. Fish intake, contaminants, and human health- Evaluating the risks and the benefits. JAMA: Journal of the American Medical Asociation 2006; 296 (15): 1885-99.

27. U.S. Department of Agriculture and U.S. Department of Health and Human Services. Dietary Guidelines for Americans, 2010. 7th Edition, Washington, DC: U.S. Government Printing Office, December 2010.

28. González C, 2004. Nuevo Reglamento Sanitario de los Alimentos. Ediciones Publiley, Santiago; 2004. pp. 63-9.

29. Israel GD. Determining Sample Size. Florida Cooperative Extension Service, University of Florida. 1992. PEOD-6. 